\title{
Supersolid phases of light in extended Jaynes-Cummings-Hubbard systems
}

\author{
B. Bujnowski, ${ }^{1}$ J. K. Corso, ${ }^{1}$ A. L. C. Hayward, ${ }^{1}$ J. H. Cole, ${ }^{2}$ and A. M. Martin ${ }^{1}$ \\ ${ }^{1}$ School of Physics, University of Melbourne, Victoria 3010, Australia \\ ${ }^{2}$ Chemical and Quantum Physics, School of Applied Sciences, RMIT University, Victoria 3001, Australia
}

(Received 24 October 2013; published 1 October 2014)

\begin{abstract}
Jaynes-Cummings-Hubbard lattices provide unique properties for the study of correlated phases as they exhibit convenient state preparation and measurement, as well as in situ tuning of parameters. We show how to realize charge density and supersolid phases in Jaynes-Cummings-Hubbard lattices in the presence of long-range interactions. The long-range interactions are realized by the consideration of Rydberg states in coupled atomcavity systems and the introduction of additional capacitive couplings in quantum-electrodynamics circuits. We demonstrate the emergence of supersolid and checkerboard solid phases, for calculations which take into account nearest-neighbor couplings, through a mean-field decoupling.
\end{abstract}

DOI: 10.1103/PhysRevA.90.043801

PACS number(s): 42.50.Pq, 67.80.kb, 32.80.Qk

\section{INTRODUCTION}

Systems in a supersolid phase possess a spontaneously formed crystalline structure along with off-diagonal longrange order which characterizes superfluidity. The investigation of supersolid phases in condensed-matter systems has been a focus of research for more than half a century [1-5]. Until recently, this effort has primarily focused on the possible realization of a supersolid phase in ${ }^{4} \mathrm{He}$ [6-8], with the most credible claim for observation [9] now being withdrawn [10]. The relatively recent realization of Bose-Einstein condensates, such as ${ }^{52} \mathrm{Cr}[11,12],{ }^{164} \mathrm{Dy}[13]$, and ${ }^{168} \mathrm{Er}$ [14], composed of atoms with large dipole moments [15], has provided an alternative avenue to investigate supersolid phases in extended Bose-Hubbard lattice models [16-21].

In this work, we investigate the emergence of charge density wave and supersolid phases in Jaynes-CummingsHubbard (JCH) lattices. Conventional JCH lattices consist of an array of coupled cavities, with each cavity mode coupled to a two-level system. A JCH system could be realized in, for example, photonic band-gap structures [22,23] and coupled-cavity waveguides [24,25], arrays of superconducting strip-line cavities [26], or microcavities with individual cold atoms connected via optical fiber interconnects [27-29]. To date, JCH systems are predicted to exhibit a number of solidstate phenomena such as superfluid and Mott-insulator phases [22-24,30-33], the Josephson effect [34], metamaterial properties [35], Bose-glass phases [25], and fractional quantum Hall physics [36]. The JCH model has recently been experimentally realized for two sites using the internal and radial phonon states of two trapped ions [37].

Through the inclusion of a long-range interaction between the two-level systems, we show that it is possible for supersolid phases to emerge in the JCH Hamiltonian. To enable the long-range interaction, we consider two cases: (i) coupled microcavities with a single atom in each cavity and (ii) arrays of superconducting strip-line cavities. For microcavities, the long-range interaction is achieved by accessing Rydberg states inducing a dipole interaction between the atoms in each cavity. For arrays of superconducting strip-line cavities, the long-range interaction is mediated via capacitive couplings within the circuit.

Previous work has shown that for a lattice of Rydberg atoms within a single cavity, a supersolid phase can emerge, where both superradiance and crystalline orders coexist [38]. In addition, driven coupled cavity systems are also predicted to result in a supersolid phase [39].

In this paper, we initially (Sec. II) focus on coupling to Rydberg states in a single cavity containing a single atom, via a two-photon process. In Sec. III, we introduce coupling between the atom cavities, mediated via both photon tunneling between cavities and dipole-dipole interactions between atoms. In Sec. IV, we then consider exact solutions for a system of four coupled atom cavities, specifically focusing on the role of nearest-neighbor interactions. We then consider, in Sec. V, mean-field solutions, demonstrating the emergence of checkerboard solid and supersolid phases in the presence of nearest-neighbor interactions.

\section{TWO-PHOTON COUPLING TO RYDBERG STATES IN A SINGLE-ATOM CAVITY}

Before considering a lattice, we focus on the properties of a single site. To achieve long-range interactions in the coupled atom-cavity system, we require the excited state of the atom to have a significant dipole moment. A possible realization of an atomic cavity that exhibits a dipole moment when excited utilizes the Rydberg state of ${ }^{87} \mathrm{Rb}$ atoms. The $5 S_{1 / 2}$ ground state $|g\rangle$ of the ${ }^{87} \mathrm{Rb}$ atom, which has been placed inside the cavity, is resonantly coupled to the Rydberg state $|e\rangle$ via a two-photon process, by using the $5 P_{3 / 2}$ state $|i\rangle$ as an intermediate step [Fig. 1(a)]. By choosing appropriate detunings for the driving fields, the intermediate state can be eliminated adiabatically as there are only small changes in its population over time. As schematically shown in Fig. 1(a), the transition from the ground state $|g\rangle$ to intermediate state $|i\rangle$ of frequency $\omega_{g i}$ is driven by the nonresonant coupling of strength $\beta$ to a single quantized cavity mode of frequency $\omega_{a}$ and photonic annihilation operator $\hat{a}^{\dagger}$, where the cavity mode is detuned by $\Delta=\omega_{a}-\omega_{g i}$. The remaining transition from $|i\rangle$ to $|e\rangle$ of frequency $\omega_{i e}$ is nonresonantly driven by a classical laser field with frequency $\omega_{l}$ and Rabi frequency $\Omega$. Transferring the interacting part of the Hamiltonian into the interaction picture and applying the rotating wave approximation, the Hamiltonian can be written in the following form $(\hbar=1)$ :

$$
\hat{\mathcal{H}}_{1}^{\text {int }}=-\Delta|i\rangle\langle i|+\left(\beta \hat{a}^{\dagger}|g\rangle\langle i|+\Omega| e\rangle\langle i|+\text { H.c. }\right) .
$$




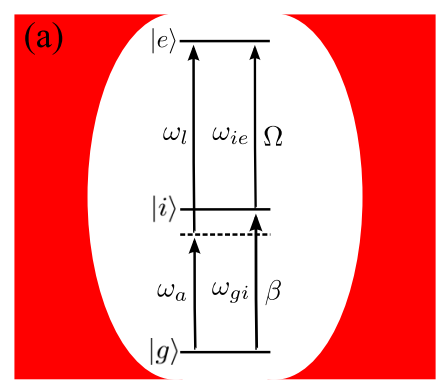

(b)

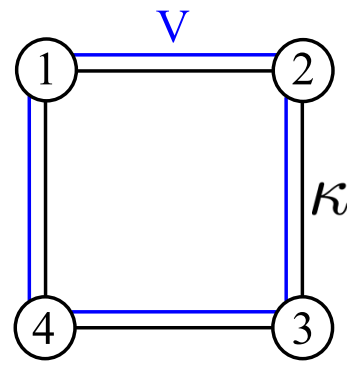

FIG. 1. (Color online) (a) Scheme of a photon cavity containing a three-level atom. The ground state $|g\rangle$ is resonantly coupled to the Rydberg state $|e\rangle$ via a two-photon process. First, the atom is excited nonresonantly by a photon of the cavity mode with frequency $\omega_{a}$. The transition to the Rydberg level happens by absorbing a photon from a driving laser field with frequency $\omega_{l}$. (b) Block of four cavities and with nearest-neighbor photon hopping $\kappa$ (black lines) and nearestneighbor interactions $V$ (blue lines).

For large detuning $\Delta$ compared to the lifetime of $|i\rangle$, the intermediate state is weakly populated and can be eliminated adiabatically. This leads to the effective Hamiltonian

$$
\hat{\mathcal{H}}^{\text {eff }}=\tilde{\beta}\left(\hat{a}^{\dagger}|g\rangle\langle e|+\hat{a}| e\rangle\langle g|\right),
$$

where $\tilde{\beta}$ denotes the rescaled coupling strength between the cavity mode and the atom. Thus the three-level atomic system is approximated by a two-level system, with the excited state of the atom exhibiting a significant dipole moment. For a detailed description of the two-cavity system, including experimentally accessible parameter regimes, see the work of Wu et al. [40].

An alternative system in which to realize the JaynesCummings-Hubbard model is circuit quantum electrodynamics (cQED) [41-46]. In a typical QED circuit, the "atomic" degree of freedom is realized via a Josephson-junction circuit providing a nonlinear set of states, the lowest two of which form an effective two-level system. The photonic degree of freedom is formed from the quantized modes of a superconducting strip-line resonator. The resulting system Hamiltonian takes on an equivalent form to that of Eq. (2). This system provides several advantages, including strong atom-photon coupling and ease of integration as the form of the effective Hamiltonian can be tailored at the circuit design stage.

\section{THE EXTENDED JAYNES-CUMMINGS-HUBBARD MODEL}

An array of coupled photon cavities that contain two-level atoms can be described by the JCH model [22-24,47-49]. In this paper, we extend the JCH to the case of Rydberg atoms by including a dipole interaction term. In the grand canonical ensemble, the Hamiltonian for this system is defined as

$$
\begin{aligned}
\hat{\mathcal{H}}= & -\kappa \sum_{\langle i, j\rangle} \hat{a}_{i}^{\dagger} \hat{a}_{j}+\tilde{\beta} \sum_{i}\left(\hat{a}_{i}^{\dagger} \hat{\sigma}_{i}+\hat{a}_{i} \hat{\sigma}_{i}^{\dagger}\right)+\sum_{i}\left(\epsilon \hat{n}_{i}^{\sigma}+\omega \hat{n}_{i}^{a}\right) \\
& +\frac{V}{2} \sum_{i} \sum_{j \neq i} \frac{\hat{n}_{i}^{\sigma} \hat{n}_{j}^{\sigma}}{\left|\mathbf{r}_{i}-\mathbf{r}_{j}\right|^{3}}-\mu \sum_{i} \hat{l}_{i} .
\end{aligned}
$$

The above Hamiltonian does not include dissipation, driving, and other nonequilibrium effects, which will be present in experiment; however, it does provide a simple equilibrium model which can be used to investigate the possibility of alternative phases in such systems. The first term describes the hopping of photons between neighboring lattice sites $i$ and $j$ with hopping amplitude $\kappa$, where $\hat{a}_{i}^{\dagger}$ and $\hat{a}_{i}$ are photon creation and annihilation operators at lattice site $i$. The second term is the on-site coupling between the photons and atoms for each site $i$ as in Eq. (2), where $\hat{\sigma}_{i}^{\dagger}=\left|e_{i}\right\rangle\left\langle g_{i}\right|$ and $\hat{\sigma}_{i}=\left|g_{i}\right\rangle\left\langle e_{i}\right|$ are atomic raising and lowering operators on site $i$, respectively. The next term defines the energy associated with the atomic and photonic degrees of freedom on each site, where $\epsilon$ is the energy of the Rydberg level and $\omega$ is the frequency of the photon mode of the cavity. Here we define the atom number operator $\hat{n}_{i}^{\sigma}=\hat{\sigma}_{i}^{\dagger} \hat{\sigma}_{i}$ that counts the atoms in the excited state and the photon number operator $\hat{n}_{i}^{a}=\hat{a}_{i}^{\dagger} \hat{a}_{i}$. The fourth term is the dipole-dipole interaction between the atoms with interaction strength $V$. It raises the total energy of the system if atoms in two or more different cavities occupy excited states. The JCH model does not conserve the number of photonic or atomic excitations. However, the total number of excitations $\sum_{i} \hat{l}_{i}=\sum_{i}\left(\hat{n}_{i}^{\sigma}+\hat{n}_{i}^{a}\right)$ is conserved. The last term in Eq. (3) specifies the total number of excitations in the system via the chemical potential $\mu$.

For the CQED case, arrays of coupled cavities can be fabricated with either capacitive or inductive coupling linking the resonators. This architecture provides an entirely equivalent realization of the JCH model [26,50]. In principle, coupling the "atoms" in a cQED system can be achieved via direct qubit-qubit coupling [51] or via the inclusion of additional components to provide an effective coupling term [52-56]. Although the exact functional form of the coupling will depend strongly on the particular circuit realization, Fig. 2 provides a schematic for two layers of a possible multiple-layer circuit. Each layer in the circuit consists of a one-dimensional array of Josephson-junction-based two-level systems coupled via

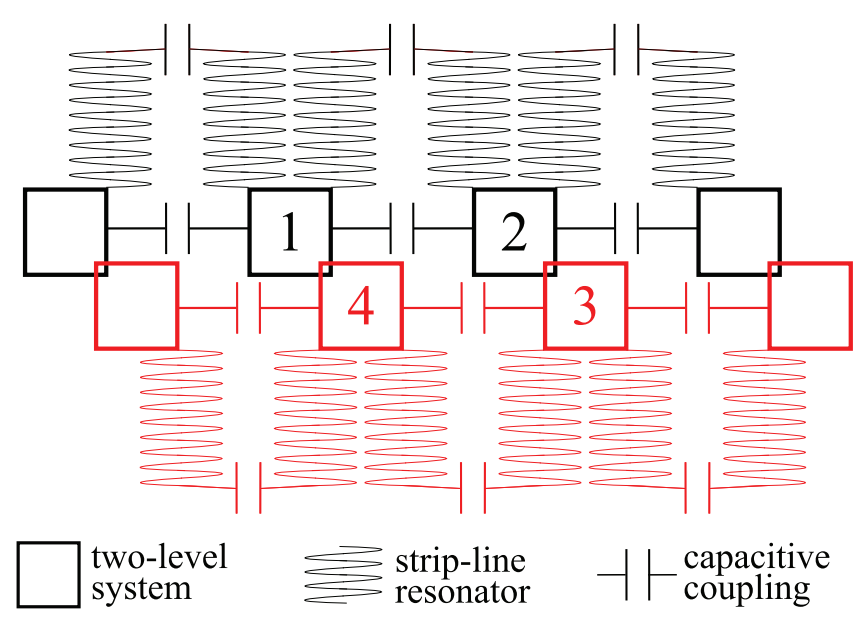

FIG. 2. (Color online) Schematic of a possible cQED realization of the long-range JCH model. Here we show two layers (black and red) of a multilayered circuit. In each layer, Josephson-junction-based two-level systems are coupled via strip-line resonators and capacitors, as denoted in the figure. Multiple layers of these one-dimensional arrays are placed on top of each other to realize an effective twodimensional (2D) lattice. 
strip-line resonators and capacitors. In such a circuit, the photonic components of the JCH model are now microwave excitations in the strip-line resonators and the long-range interactions (in this case, nearest neighbor) arise from capacitive coupling between adjacent Josephson-junction two-level systems. For a multilayered system, capacitive coupling between strip-line resonators in adjacent layers enables microwave excitations to couple between layers. Additionally, capacitive coupling between Josephson junctions in adjacent layers mediates a long-range interaction between two-level systems. Multiple layers where coupling between strip-lines and Josephson junctions is only between adjacent layers and nearest neighbors form an effective two-dimensional lattice. A useful variant is to use "flux qubits" and $L C$ resonators for the atomic and photonic components, respectively, as this results in a smaller circuit footprint $[57,58]$.

For the coupled atom-cavity system, the dipole interactions decay as $\left|\mathbf{r}_{i}-\mathbf{r}_{j}\right|^{-3}$; see second-to-last term in Eq. (3). For the cQED case, the exact parametrization of the long-range interactions depends on the capacitive couplings between qubits, with the exact coupling defined by the circuit geometry. The aim of this work is to show that for either a coupled atom-cavity system or the cQED case, extended interactions will lead to alternative charge density wave and supersolid phases. As such, we consider the simplest form of extended interactions (nearest neighbor), with the resulting Hamiltonian

$$
\begin{aligned}
\hat{\mathcal{H}}= & -\kappa \sum_{\langle i, j\rangle} \hat{a}_{i}^{\dagger} \hat{a}_{j}+\tilde{\beta} \sum_{i}\left(\hat{a}_{i}^{\dagger} \hat{\sigma}_{i}+\hat{a}_{i} \hat{\sigma}_{i}^{\dagger}\right)+\sum_{i}\left(\epsilon \hat{n}_{i}^{\sigma}+\omega \hat{n}_{i}^{a}\right) \\
& +\frac{V}{2} \sum_{\langle i, j\rangle} \hat{n}_{i}^{\sigma} \hat{n}_{j}^{\sigma}-\mu \sum_{i} \hat{l}_{i} .
\end{aligned}
$$

\section{FOUR-SITE SOLUTIONS OF THE EXTENDED JAYNES-CUMMINGS-HUBBARD MODEL}

In Sec. V, we will consider mean-field solutions for an infinite array of coupled atom cavities. The mean-field approximation will be based on the coupling of a four-site system to an infinite lattice. As such, before proceeding with the mean-field coupling, we first consider the exact solutions of a four-site system. The system under consideration is schematically shown in Fig. 1(b), i.e., four atom cavities in a square arrangement, with nearest-neighbor hopping and nearest-neighbor interactions. For such a system, the Hamiltonian is

$$
\begin{aligned}
\hat{\mathcal{H}}_{4}= & -z \bar{\kappa}\left(\hat{a}_{1}^{\dagger} \hat{a}_{2}+\hat{a}_{2}^{\dagger} \hat{a}_{1}+\hat{a}_{2}^{\dagger} \hat{a}_{3}+\hat{a}_{3}^{\dagger} \hat{a}_{2}\right. \\
& \left.+\hat{a}_{3}^{\dagger} \hat{a}_{4}+\hat{a}_{4}^{\dagger} \hat{a}_{3}+\hat{a}_{1}^{\dagger} \hat{a}_{4}+\hat{a}_{4}^{\dagger} \hat{a}_{1}\right) \\
& +\sum_{i=1}^{4}\left[-\bar{\mu} \hat{n}_{i}^{a}-(\bar{\Delta}+\bar{\mu}) \hat{n}_{i}^{\sigma}+\left(\hat{\sigma}_{i}^{\dagger} \hat{a}_{i}+\hat{a}^{\dagger} \hat{\sigma}_{i}\right)\right] \\
& +z \bar{V}\left(\hat{n}_{1}^{\sigma} \hat{n}_{2}^{\sigma}+\hat{n}_{2}^{\sigma} \hat{n}_{3}^{\sigma}+\hat{n}_{3}^{\sigma} \hat{n}_{4}^{\sigma}+\hat{n}_{4}^{\sigma} \hat{n}_{1}^{\sigma}\right),
\end{aligned}
$$

where $z=1(z=2)$ for fixed (periodic) boundary conditions. In the above, we have introduced the following dimensionless couplings: $\bar{\kappa}=\kappa / \tilde{\beta}, \bar{\mu}=(\mu-\omega) / \tilde{\beta}, \bar{\Delta}=(\omega-\epsilon) / \tilde{\beta}$, and $\bar{V}=V /\left(\tilde{\beta} l_{0}\right)$, where $l_{0}$ is the lattice unit length. Note that switching between periodic and fixed boundary conditions can be included by a trivial rescaling of $\bar{\kappa}$ and $\bar{V}$.

To find the ground state of the four-site system, we evaluate Eq. (5) in the following basis: $\left\{\prod_{i=1}^{4}\left|n_{i}^{a}, n_{i}^{\sigma}\right\rangle ; n_{i}^{a} \in \mathbb{N}_{0}, n_{i}^{\sigma} \in\right.$ $\{0,1\}\}$. Due to computational limitations, it is necessary to restrict the possible basis states. In the following, a cutoff defined by $n_{i}^{a}+n_{i}^{\sigma} \leqslant 5$ was used on every site, which is more than sufficient to demonstrate the fundamental ground-state properties of the four-site system.

Figure 3 plots the number of excitations per site $(n=$ $\left.\frac{1}{4} \sum_{i=1}^{4}\left\langle\hat{l}_{i}\right\rangle\right)$, in the ground state, for the four-site system, with periodic boundary conditions $(z=2)$ as a function of the chemical-potential and photon-hopping strength, for various strengths of nearest-neighbor interactions, with $\bar{\Delta}=0$. In the absence of dipolar interactions $(\bar{V}=0)$, we observe a pinch effect as $\bar{\kappa} \rightarrow 0$ between $n=k$ and $n=k+1$, where $k=$ $1,2,3, \ldots$, i.e., all fractional occupations disappear as $\bar{\kappa} \rightarrow 0$, consistent with previous results. This implies that in the limit of no photon hopping between the cavities, the ground state is defined by the number of excitations being an integer multiple of the number of sites. As photon hopping is introduced, states emerge with the number of excitations not being an integer multiple of the number of sites. Introducing nearest-neighbor interactions significantly changes the ground-state properties
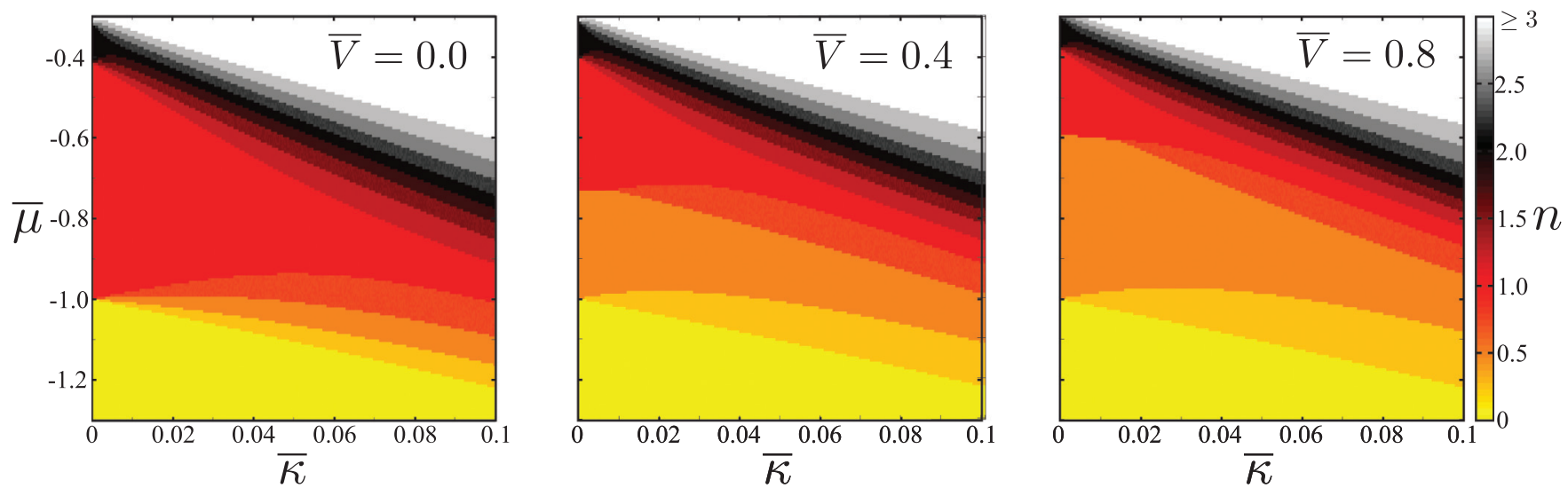

FIG. 3. (Color online) Number of excitations per site, $n=\frac{1}{4} \sum_{i=1}^{4}\left\langle\hat{l}_{i}\right\rangle$, in a system of four coupled atom cavities, with periodic boundary conditions, as a function of the chemical potential $\bar{\mu}$ and the intercavity hopping $\bar{\kappa}$, for different values of the nearest-neighbor interaction $\bar{V}$, with $\bar{\Delta}=0$. 
of the system. Specifically, we see that as $\bar{\kappa} \rightarrow 0$, fractional occupations do not disappear, i.e., for $\bar{\kappa} \rightarrow 0$, a state emerges with $n=0.5$. This corresponds to a checkerboard phase with atomic excitations arranged on the diagonal to minimize the nearest-neighbor interaction. As the strength of the nearest-neighbor interaction increases, the chemical-potential range over which this fractional state exists grows. For the fractional $n=0.5$ state, in the limit $\bar{\kappa} \rightarrow 0$, we find the the atomic and photonic contributions to this state are equal, i.e., $\sum_{i=1}^{4}\left\langle\hat{n}_{i}^{\sigma}\right\rangle=\sum_{i=1}^{4}\left\langle\hat{n}_{i}^{a}\right\rangle=1$. Additionally, for the $n=1$ and $n=2$ states, the introduction of nearest-neighbor interactions increases (decreases) the photonic (atomic) contributions to the number of excitations per site. The emergence of the $n=0.5$ checkerboard state corresponds to a checkerboard solid. The increase (decrease) in the photonic (atomic) contributions to the number of excitations per site (for $n=1$ and $n=2$ ) comes from the energy cost of having atomic excitations in the system, due to the nearest-neighbor interactions.

\section{MEAN-FIELD SOLUTIONS OF THE EXTENDED JAYNES-CUMMINGS-HUBBARD MODEL}

For the case of vanishing long-range interaction $(\bar{V}=$ 0 ), phase transitions between homogeneous superfluid and homogeneous Mott-insulator phases [22-24,30,31] have been predicted. To determine these phases, one approach is to introduce a mean-field decoupling in the hopping term, between the lattice sites. For a single-site decoupling, this restricts the excitations to be homogeneously distributed.

Including the nearest-neighbor interactions $(\bar{V} \neq 0)$, we expect a nonhomogeneous distribution of excitations due to the energy cost of having more than one atom in the excited state close to each other. To observe this in the mean-field model, we allow the order parameters to vary across the system. For interactions between nearest-neighbors only, variations of the order parameter appear with a maximum period of two lattice unit lengths. Thus it is sufficient to decouple the infinite square lattice of cavities into periodical square blocks of four, as depicted in Fig. 4. Tunneling (dashed lines) and long-range interactions between blocks (dotted lines) are treated by a meanfield approximation, i.e., operator products that connect two blocks are approximated by $\hat{A} \hat{B} \approx \hat{A}\langle\hat{B}\rangle+\langle\hat{A}\rangle \hat{B}-\langle\hat{A}\rangle\langle\hat{B}\rangle$. Intrablock tunneling and long-range interactions (solid lines) are kept to capture the significant effects of correlations within the block. The approximated Hamiltonian can be written as a sum of terms within the block and mean-field terms connecting the block to the surrounding lattice sites, $\hat{\mathcal{H}} \approx \hat{\mathcal{H}}^{\prime}=\hat{\mathcal{H}}_{4}+\hat{\mathcal{H}}_{\mathrm{MF}}$, where $\hat{\mathcal{H}}_{4}$ is given by Eq. (5), with $z=1$, and $\hat{\mathcal{H}}_{\mathrm{MF}}=\hat{\mathcal{H}}_{\mathrm{MF}}^{12}+\hat{\mathcal{H}}_{\mathrm{MF}}^{23}+\hat{\mathcal{H}}_{\mathrm{MF}}^{34}+\hat{\mathcal{H}}_{\mathrm{MF}}^{43}$ with

$$
\begin{aligned}
\hat{\mathcal{H}}_{\mathrm{MF}}^{i j}= & -\bar{\kappa}\left\{\left(\hat{a}_{i}^{\dagger}+\hat{a}_{i}\right) \psi_{j}+\left(\hat{a}_{j}^{\dagger}+\hat{a}_{j}\right) \psi_{i}-2 \psi_{i} \psi_{j}\right\} \\
& +\bar{V}\left\{\hat{n}_{i} \xi_{j}+\hat{n}_{j} \xi_{i}-\xi_{i} \xi_{j}\right\} .
\end{aligned}
$$

Here we introduced the mean-field parameter of the atomic excitation on each site $\xi_{i}=\left\langle\hat{n}_{i}^{\sigma}\right\rangle$ and the real superfluid order parameter $\psi_{i}=\left\langle\hat{a}_{i}\right\rangle$.

To find the ground state of $\hat{\mathcal{H}}^{\prime}$, we calculate the order parameters self-consistently, minimizing the total energy of the system simultaneously. To avoid convergence problems, due to degenerate ground states in a nonhomogeneous phase,

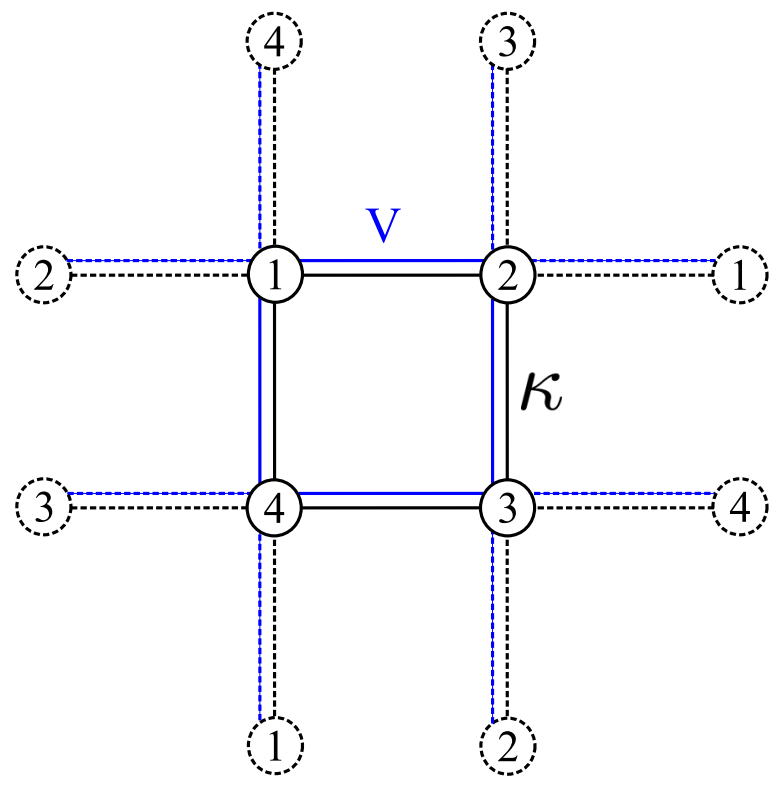

FIG. 4. (Color online) Scheme of the mean-field approximation for a block of four cavities and nearest-neighbor interactions. The intrablock hopping $\kappa$ (black solid line) and nearest-neighbor dipole interactions $V$ (blue solid line) between neighboring cavities (black circles) are treated identically. Interactions outside of the block are decoupled by a mean-field approximation (black and blue dashed lines).

the symmetry of the block is broken by introducing a random energy shift of $\delta_{i}$ for each cavity. This energy shift [59], in dimensionless units $\bar{\delta}_{i}=\delta_{i} / \beta$, is of the order of $10^{-6}$. As in Sec. IV, the basis is restricted to $n_{i}^{a}+n_{i}^{\sigma} \leqslant 5$ on each site.

The phase diagram for our system can be deduced from Figs. 5 and 6. For $\bar{V}=0$, we find the well-known result that the parameter space is separated into two distinct phases. For low hopping strength $\bar{\kappa}$, we find lobes of vanishing superfluid order parameter, i.e., Mott-insulating phases as shown in Fig. 6(a). Each lobe corresponds to a state with an integer number of strongly localized excitations per site. This is shown in Fig. 5, where we plot the mean number of excitations per site in the Mott-insulator phase. For low chemical potential, there are no excitations in the system. Raising the chemical potential, the block is successively filled with one, two, and more excitations per site. At sufficiently large $\bar{\kappa}$, the system undergoes a phase transition into a phase of finite superfluid order parameter [Fig. 6(a)]. The excitations are homogeneously distributed and delocalized, i.e., the system is in a superfluid state.

Consistent with the finite-size results (Fig. 3), Fig. 5 shows that as $\bar{V}$ is increased from zero, a phase emerges with $n=1 / 2$ and a zero supersolid or superfluid component, corresponding to two excitations per block. This corresponds to a checkerboard solid phase with excitations arranged on the diagonal to minimize the nearest-neighbor interaction. As the strength of the nearest-neighbor interactions are increased, the extent of this checkerboard solid phase increases. Checkerboard solid phases also appear at higher filling $\left(n=\frac{3}{2}, n=\frac{5}{2}\right)$. However, for the values of $\bar{V}$ considered, the extent of phases is very small. 

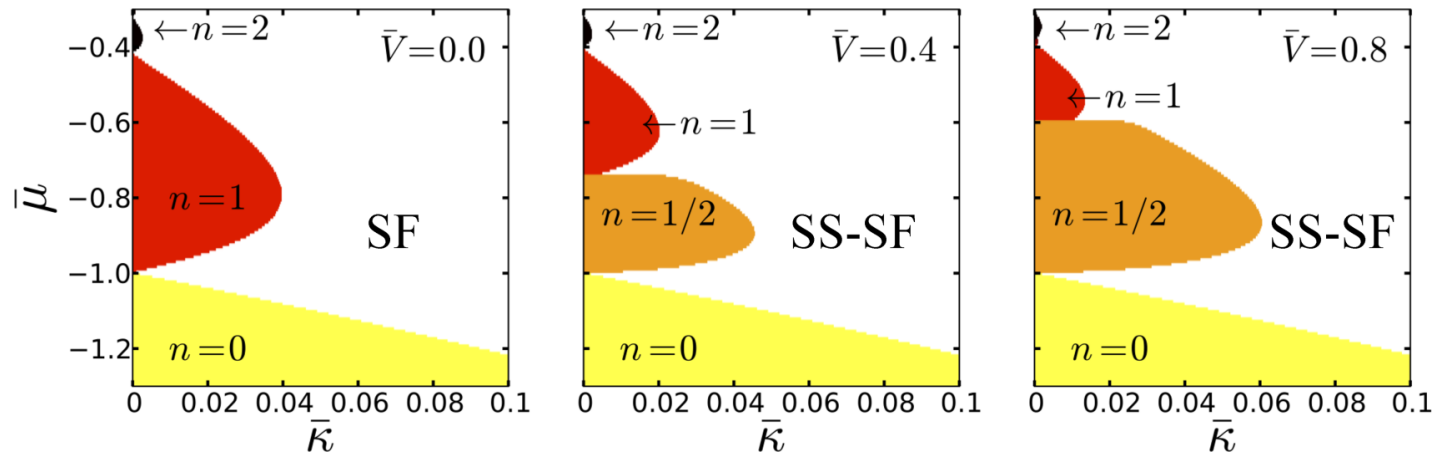

FIG. 5. (Color online) Mean number of excitations per block of four cavities $n=\frac{1}{4} \sum_{i=1}^{4}\left\langle\hat{l}_{i}\right\rangle$ within the Mott-insulator and checkerboard phases, as a function of the chemical potential $\bar{\mu}$ and the intercavity hopping $\bar{\kappa}$, for different values of the nearest-neighbor interaction $\bar{V}$, with $\bar{\Delta}=0$. In the white supersolid-superfluid (SS-SF) region, we find nonvanishing superfluid order parameters, i.e., $\sum_{i=1}^{4} \psi_{i}>0$.

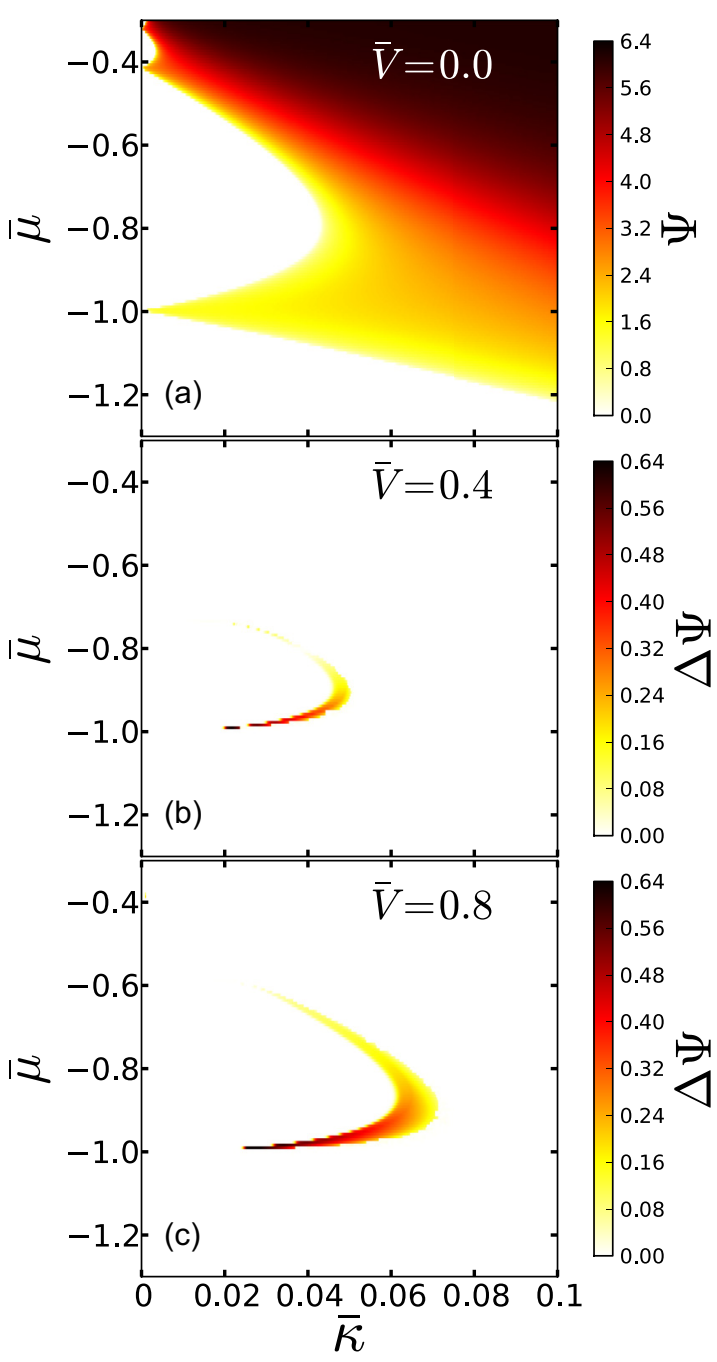

FIG. 6. (Color online) (a) The sum of the superfluid order parameters on each block $\Psi=\sum_{i=1}^{4} \psi_{i}$ as a function of the chemical potential $\bar{\mu}$ and the intercavity hopping $\bar{\kappa}$ for $\bar{V}=0.0$. (b),(c) $\Delta \Psi=\left|\psi_{1}-\psi_{2}\right| /\left|\psi_{1}+\psi_{2}\right|$ as a function of the chemical potential $\bar{\mu}$ and the intercavity hopping $\bar{\kappa}$ for $\bar{V}=0.4$ and 0.8 . This normalized difference between the order parameters indicates the presence of a checkerboard supersolid (SS) phase.
Associated with the emergence of the checkerboard solid phase is supersolid behavior. Supersolid regimes can be characterized by identifying changes in the superfluid order parameters within the block. In the absence of nearestneighbor interactions, $\psi_{1}=\psi_{2}=\psi_{3}=\psi_{4}$. However, for $\bar{V}>0$, we find regimes where $\psi_{1}=\psi_{3}$ and $\psi_{2}=\psi_{4}$ but $\psi_{1} \neq \psi_{2}$ corresponding to a supersolid phase. Figures 6(b) and 6(c) identify the supersolid phase by plotting $\Delta \Psi=$ $\left|\psi_{1}-\psi_{2}\right| /\left|\psi_{1}+\psi_{2}\right|$, in the region where $\sum_{i=1}^{4} \psi_{i}>0$. As can be seen from Fig. 5, this supersolid phase is present at the interface of the checkerboard solid phase and as $\bar{\kappa}$ is increased, it diminishes, until $\psi_{1}=\psi_{2}$, characterizing a superfluid state. As for the checkerboard solid phase, as $\bar{V}$ is increased, the extent of the supersolid phase increases.

Intrablock correlations play a crucial role in determining the phase diagram in the extended $\mathrm{JCH}$ model. Introducing mean-field decouplings within the block for the hopping and nearest-neighbor interactions [first and last terms in Eq. (5)] changes the phase diagram for $\bar{V} \neq 0$. Decoupling results in a decrease of the extent of the checkerboard solid and supersolid phases. These shifts grow in significance when $\bar{V} \rightarrow 1$. This is in contrast to the case $\bar{V}=0$ where the phase diagram for a single-site decoupling [22-24,31] and the blockwise decoupling does not differ significantly. Additionally, for supersolid phases in the extended Bose-Hubbard model, it has been found that quantum fluctuations can play a significant role in determining stability [18]. As such, to test the robustness of these mean-field calculations, quantum Monte Carlo methods should be employed.

\section{CONCLUSIONS}

The nature of the nearest-neighbor interaction in the $\mathrm{JCH}$ model is qualitatively different from that found in other lattice systems with long-range interactions, such as ultracold dipolar gases in optical lattices, where the extended Bose-Hubbard model is appropriate. Specifically, in the extended JCH model, the interaction is mediated via a two-level system. Thus the interaction depends on the simultaneous excitation of neighboring atoms, which favors antiferromagnetic correlations between the atomic states. Indeed, at $\kappa=0$, the $\mathrm{JCH}$ system 
maps to a quantum Heisenberg model [24,60-62], in contrast to the Bose-Hubbard case, which lies in the classical Ising universality class.

We have demonstrated that the inclusion of long-range interactions in the $\mathrm{JCH}$ model results in the emergence of (i) noninteger Mott-insulator phases and (ii) supersolid phases. In the absence of long-range interactions, the two-level systems mediate an interaction between photons in the lattice. The predicted Mott-insulator superfluid transition is a direct consequence of this interaction. The addition of a direct coupling between the two-level systems introduces charge density and supersolid phases. Such an extended interaction can be mediated in coupled atom-cavity systems through the inclusion of Rydberg states and in CQED systems via capacitive couplings between qubits. JCH systems provide an alternative platform to investigate the emergence of supersolid phases and novel correlated states of light.

Experimental realizations of the JCH model are subject to loss mechanisms, which are not included in the above work. As such, the analysis presented above is valid for regimes where the quality factor of the cavities is large and the hopping rates between the cavities dominates over absorption or loss of photons out of the system. To study the robustness of the charge density and supersolid phases for systems away from this regime would require the inclusion of both driving and dissipation in the Hamiltonian [63].
[1] E. P. Gross, Phys. Rev. 106, 161 (1957).

[2] A. F. Andreev and I. M. Lifshitz, Zh. Eksp. Theor. Fiz. 56, 2057 (1969) [Sov. Phys. JETP 29, 1107 (1969)].

[3] D. J. Thouless, Ann. Phys. 52, 403 (1969).

[4] A. J. Leggett, Phys. Rev. Lett. 25, 1543 (1970).

[5] G. V. Chester, Phys. Rev. A 2, 256 (1970).

[6] S. Balibar, A. D. Fefferman, A. Haziot, and X. Rojas, J. Low Temp. Phys. 168, 221 (2012).

[7] P. Nozières, J. Low Temp. Phys. 142, 91 (2006).

[8] P. Nozières, J. Low Temp. Phys. 156, 9 (2009).

[9] E. Kim and M. H. W. Chan, Nature (London) 427, 225 (2004); Science 305, 1941 (2004).

[10] D. Y. Kim and M. H. W. Chan, Phys. Rev. Lett. 109, 155301 (2012).

[11] A. Griesmaier, J. Werner, S. Hensler, J. Stuhler, and T. Pfau, Phys. Rev. Lett. 94, 160401 (2005).

[12] Q. Beaufils, R. Chicireanu, T. Zanon, B. Laburthe-Tolra, E. Maréchal, L. Vernac, J.-C. Keller, and O. Gorceix, Phys. Rev. A 77, 061601(R) (2008).

[13] M. Lu, N. Q. Burdick, S. H. Youn, and B. L. Lev, Phys. Rev. Lett. 107, 190401 (2011).

[14] K. Aikawa, A. Frisch, M. Mark, S. Baier, A. Rietzler, R. Grimm, and F. Ferlaino, Phys. Rev. Lett. 108, 210401 (2012).

[15] T. Lahaye, C. Menotti, L. Santos, M. Lewenstein, and T. Pfau, Rep. Prog. Phys. 72, 126401 (2009).

[16] K. Góral, L. Santos, and M. Lewenstein, Phys. Rev. Lett. 88, 170406 (2002).

[17] D. L. Kovrizhin, G. V. Pai, and S. Sinha, Europhys. Lett. 72, 162 (2005).

[18] P. Sengupta, L. P. Pryadko, F. Alet, M. Troyer, and G. Schmid, Phys. Rev. Lett. 94, 207202 (2005).

[19] V. W. Scarola and S. Das Sarma, Phys. Rev. Lett. 95, 033003 (2005).

[20] V. W. Scarola, E. Demler, and S. Das Sarma, Phys. Rev. A 73, 051601(R) (2006).

[21] E. G. Dalla Torre, E. Berg, and E. Altman, Phys. Rev. Lett. 97, 260401 (2006)

[22] M. J. Hartmann, F. G. S. L. Brandão, and M. B. Plenio, Nat. Phys. 2, 849 (2006).

[23] A. D. Greentree, C. Tahan, J. H. Cole, and L. C. L. Hollenberg, Nat. Phys. 2, 856 (2006).

[24] D. G. Angelakis, M. F. Santos, and S. Bose, Phys. Rev. A 76, 031805(R) (2007).
[25] D. Rossini and R. Fazio, Phys. Rev. Lett. 99, 186401 (2007).

[26] M. Leib and M. J. Hartmann, New J. Phys. 12, 093031 (2010)

[27] M. Trupke, E. A. Hinds, S. Eriksson, E. A. Curtis, and Z. Moktadir, Appl. Phys. Lett. 87, 211106 (2005).

[28] I. Bloch, Nat. Phys. 1, 23 (2005).

[29] R. Balili, V. Hartwell, D. Snoke, L. Pfeiffer, and K. West, Science 316, 1007 (2007).

[30] S. Schmidt and G. Blatter, Phys. Rev. Lett. 103, 086403 (2009).

[31] J. Koch and K. Le Hur, Phys. Rev. A 80, 023811 (2009).

[32] S. Schmidt and G. Blatter, Phys. Rev. Lett. 104, 216402 (2010).

[33] A. Tomadin, V. Giovannetti, R. Fazio, D. Gerace, I. Carusotto, H. E. Türeci, and A. Imamoglu, Phys. Rev. A 81, 061801(R) (2010).

[34] S.-C. Lei, T.-K. Ng, and R.-K. Lee, Opt. Express 18, 14586 (2010).

[35] J. Q. Quach, C.-H. Su, A. M. Martin, A. D. Greentree, and L. C. L. Hollenberg, Opt. Express 19, 11018 (2011).

[36] A. L. C. Hayward, A. M. Martin, and A. D. Greentree, Phys. Rev. Lett. 108, 223602 (2012).

[37] K. Toyoda, Y. Matsuno, A. Noguchi, S. Haze, and S. Urabe, Phys. Rev. Lett. 111, 160501 (2013).

[38] X. F. Zhang, Q. Sun, Y. C. Wen, W.-M. Liu, S. Eggert, and A.-C. Ji, Phys. Rev. Lett. 110, 090402 (2013).

[39] J. Jin, D. Rossini, R. Fazio, M. Leib, and M. J. Hartmann, Phys. Rev. A 90, 023827 (2014).

[40] H. Wu, Z.-B. Yang, and S.-B. Zheng, Phys. Rev. A 88, 043816 (2013).

[41] A. Blais, R.-S. Huang, A. Wallraff, S. M. Girvin, and R. J. Schoelkopf, Phys. Rev. A 69, 062320 (2004).

[42] A. Wallraff, D. I. Schuster, A. Blais, L. Frunzio, R. S. Huang, J. Majer, S. Kumar, S. M. Girvin, and R. J. Schoelkopf, Nature (London) 431, 162 (2004).

[43] D. I. Schuster, A. A. Houck, J. A. Schreier, A. Wallraff, J. M. Gambetta, A. Blais, L. Frunzio, J. Majer, B. Johnson, M. H. Devoret, S. M. Girvin, and R. J. Schoelkopf, Nature (London) 445, 515 (2007).

[44] J. Li, M. P. Silveri, K. S. Kumar, J.-M. Pirkkalainen, A. Vepsäläinen, W. C. Chien, J. Tuorila, M. A. Sillanpää, P. J. Hakonen, E. V. Thuneberg, and G. S. Paraoanu, Nat. Commun. 4, 1420 (2013).

[45] J. Rafterty, D. Sadri, S. Schmidt, H. E. Türeci, and A. A. Houck, arXiv:1312.2963 (2013).

[46] S. Schmidt and J. Koch, Ann. Phys. 525, 395 (2013). 
[47] M. Knap, E. Arrigoni, and W. von der Linden, Phys. Rev. B 81, 104303 (2010).

[48] M. Knap, E. Arrigoni, W. von der Linden, and J. H. Cole, Phys. Rev. A 83, 023821 (2011).

[49] M. Knap, E. Arrigoni, and W. von der Linden, Comp. Phys. Commun. 182, 2036 (2011).

[50] M. Leib, F. Deppe, A. Marx, R. Gross, and M. J. Hartmann, New J. Phys. 14, 075024 (2012).

[51] Y. Makhlin, G. Schön, and A. Shnirman, Rev. Mod. Phys. 73, 357 (2001).

[52] G. S. Paraoanu, Phys. Rev. B 74, 140504 (2006).

[53] A. G. Fowler, W. F. Thompson, Z. Yan, A. M. Stephens, B. L. T. Plourde, and F. K. Wilhelm, Phys. Rev. B 76, 174507 (2007).

[54] J. Li, K. Chalapat, and G. S. Paraoanu, Phys. Rev. B 78, 064503 (2008).

[55] K. Harrabi, F. Yoshihara, A. O. Niskanen, Y. Nakamura, and J. S. Tsai, Phys. Rev. B 79, 020507(R) (2009).
[56] S. Ashhab, A. O. Niskanen, K. Harrabi, Y. Nakamura, T. Picot, P. C. de Groot, C. J. P. M. Harmans, J. E. Mooij, and F. Nori, Phys. Rev. B 77, 014510 (2008).

[57] I. Chiorescu, P. Bertet, K. Semba, Y. Nakamura, C. J. P. M. Harmans and J. E. Mooij, Nature (London) 431, 159 (2004).

[58] A. A. Abdumalikov Jr., O. Astafiev, Y. Nakamura, Y. A. Pashkin, and J. S. Tsai, Phys. Rev. B 78, 180502(R) (2008).

[59] This on-site energy is chosen to be small enough such that the results are independent of the particular value.

[60] M. J. Hartmann, F. G. S. L. Brandão, and M. B. Plenio, Phys. Rev. Lett. 99, 160501 (2007).

[61] J. Cho, D. G. Angelakis, and S. Bose, Phys. Rev. A 78, 062338 (2008).

[62] A. Kay and D. G. Angelakis, Europhys. Lett. 84, 20001 (2008).

[63] J. Ruiz-Rivas, E. del Valle, C. Gies, P. Gartner, and M. J. Hartmann, Phys. Rev. A 90, 033808 (2014). 